\title{
Statistical Constraints on State Preparation for a Quantum Computer
}

\author{
Subhash Kak \\ Department of Electrical \& Computer Engineering \\ Louisiana State University \\ Baton Rouge, LA 70803-5901, USA
}

March 26, 2001

\begin{abstract}
Quantum computing algorithms require that the quantum register be initially present in a superposition state. To achieve this, we consider the practical problem of creating a coherent superposition state of several qubits. We show that the constraints of quantum statistics require that the entropy of the system be brought down when several independent qubits are assembled together. In particular, we have: (i) not all initial states are realizable as pure states; (ii) the temperature of the system must be reduced. These factors, in addition to decoherence and sensitivity to errors, must be considered in the implementation of quantum computers.
\end{abstract}

Keywords. Quantum computing; initializing a quantum state PACS No. 03.65.Bz 


\section{Introduction}

There is a duality between the classical and the quantum: In the classical world objects are distinct, while in the quantum world they are indistinguishable. We, the users of quantum computing, belong to the classical world. This creates a conundrum for starting a computation on a quantum computer. How do we load information on the quantum register if the information-carrying particles in the cells of the register are indistinguishable?

Quantum computing algorithms as visualized now $[1,2]$ proceed with the register of $n$ cells in a pure state. Each cell is seen to store a qubit $\alpha e^{i \theta_{1}}|0\rangle+\beta e^{i \theta_{2}}|1\rangle$, where $\alpha, \beta$ are real numbers and $\alpha^{2}+\beta^{2}=1$. Normally, the state of the register, $|\phi\rangle$, is taken to be the all-zero state of $n$-qubits: $|0\rangle|0\rangle \ldots|0\rangle$, or the amplitude vector $(1,0,0 \ldots 0)$, which, by a process of rotation transformations on each qubit, is transformed into the state with ampli-

tudes $\left(\frac{1}{\sqrt{N}}, \frac{1}{\sqrt{N}}, \frac{1}{\sqrt{N}} \ldots \frac{1}{\sqrt{N}}\right)$, where $N=2^{n}$. In the most general case, the state function can be written out as:

$$
|\phi\rangle=\sum_{x=00 \ldots 0}^{11 \ldots 1} c_{x}|x\rangle
$$

where the $c_{x}$ are complex numbers $\left(\sum\left|c_{x}\right|^{2}=1\right)$ and the index $x$ ranges over all $2^{n}$ values of an $n$-bit string. Quantum computing is the application of appropriate unitary transformations on an initial state function that describes the problem to be computed.

Implementation issues related to decoherence and sensitivity to errors, after the computation has started, have been considered in the literature [3]. In particular, several groups [4] have used trapped ion and NMR techniques to run simple quantum algorithms. But they have not demonstrated the initialization of an arbitrary pure state in the register. It has been shown [5], that the initial state loaded in these examples was a mixture, and so these early efforts do not fully implement eqn. (1). The NMR experiments are thus to be properly seen as simulations of quantum computations rather than true quantum computations.

There exist several interesting problems with the model of eqn. (1) regarding manipulations of the contents of a quantum register. The assumption inherent in the algorithms that the phase uncertainty related to the $c_{x}$ s in 
each of these superposition states is identical and it can be lumped together and ignored has been questioned [6]. It has also been shown that it is impossible to delete an unknown quantum state. In particular, if there are several copies of an unknown photon, it is not possible to delete the information content of one or more of these photons by a physical process [7]. Likewise, it is not always possible to go from one state to another using local transformations [8]. These problems arise due to the nature of superpositions in a quantum state.

The problem related to the difficulty of the transformation of one quantum state to another may be posed in other forms as well. Here we consider the question of starting from a suitable initial state on the register to reach the amplitude vector $\left(\frac{1}{\sqrt{N}}, \frac{1}{\sqrt{N}}, \frac{1}{\sqrt{N}} \cdots \frac{1}{\sqrt{N}}\right)$, a superposition of all the components. We show that statistical constraints make it impossible to do so under ordinary circumstances. However, we do not argue that it is impossible, in principle, to prepare such a state.

\section{Preparing the superposition state}

Consider a quantum register with $n$ cells, each containing a qubit. If the qubits are independent quantum systems, and they are brought together, we will have a mixture. We can see this clearly by imagining that the individual qubits are physically remote from each other.

The challenge is to obtain a superposition state that is coherent so that it can be considered a single quantum system. Qubits, generated separately and assembled together, do not create the appropriate superposition, because this assembly ignores the constraints of quantum statistics. The process of bringing the qubits together alters the conditions related to the preparation of the qubits. So how do the distinguishable particles which are the starting qubits make a transition to the superposition state of the collection?

Before, answering this question, one must note that the use of classical notions in considering the contents of the register can lead to erroneous conclusions. For a quantum system, it is essential to speak not in terms of $a$ priori properties, but in terms of state preparation and observation. Quantum mechanics is not a theory about reality; it is a prescription for making the best possible predictions about the future, if we have certain information about the past [9]. 
A pure state is one which yields a specific outcome in a given test designed to elicit the maximum number of outcomes associated with the system [6]. Examples are Stern-Gerlach experiment for spin or the use of a calcite crystal for photon polarization. One may represent the all-zero state by $(1,0,0 \ldots 0)$, if it is taken that each of the qubits has been prepared identically and there is no dynamical evolution. This is equivalent to considering that qubits emerging out of the state preparation apparatus are frozen in their state and installed at the appropriate locations in the quantum register.

If the particles are generated at a certain point and tested to yield a specific outcome and, if they pass the test, transported to the right locations on the register, there is no way to guarantee that each of the particles would not have suffered dynamic evolution prior to installation. If instead, $n$ tests are performed simultaneously on the particles so as to cut down on the delay, it may happen that some of these tests do not yield the specified outcome and so the failed tests will have to be repeated resulting in delay.

It appears more reasonable to assume that the qubits are already available at the cell-sites of the quantum register. These qubits then will be individually steered to a specific pure state. This assumption is actually made in the description of the process to obtain the superposition state required at the start of the quantum algorithm.

\section{Collection of qubits}

But, having done this, it is necessary to examine the collection of the $n$ qubits from a statistical perspective. As a quantum system, the register cannot be viewed as consisting of unique particles, which is what is implicit in the individual rotation of the qubits to obtain the superposition state of $2^{n}$ components. We must remember that not every pure state is realizable.

Only classical particles in a $n$-cell register, each with two states, can be distinguished in terms of $2^{n}$ total components. Quantum particles are indistinguishable and this restricts the number of possible distinct states. There will be the usual symmetry restrictions associated with the state function of the register depending on the particles being bosons or fermions.

We wish to stress the issue of indistinguishability of particles in a superposition state. For example, a $n$-cell register with polarized photons in each

cell can have only $n+1$ distinguishable states. If only one of the photons out 
of $n$ is polarized vertically, then this particular photon is not to be localized to a specific cell of the register. Although the measurement apparatus will localize the vertically polarized photon at one of the $n$-locations, this vertical polarization will be shared by all the $n$-cells and so its appearance at a specific location must be viewed in a probabilistic sense as a part of the state reduction process.

To see this further, consider a register of 3-cells where one of the three qubits is in the state $|1\rangle$ and the other two are in the state $|0\rangle$. Since the particles are indistinguishable, it is incorrect to write the state of the register as $|100\rangle$, where it is assumed that the first cell has a $|1\rangle$ qubit and the other two have $|0\rangle$ qubits. Just like an electron cannot be localized in a box before it has been observed, a specific quantum property cannot be localized to a particle that belongs to a collection. The correct state description for this case is:

$$
|\phi\rangle=\frac{1}{\sqrt{3}}|100\rangle+\frac{1}{\sqrt{3}}|010\rangle+\frac{1}{\sqrt{3}}|001\rangle
$$

where the relative phases have been ignored.

A quantum register will still yield $n$-bits of information. But the indistinguishability of the particles throws a veil over the quantum reality which limits our capacity to structure the states on the register and to speak of a specific characteristic of the particles in the set.

It is indeed true that the atoms at different locations, carrying qubits, are physically distinct. But such distinct qubits are normally in a separable, mixed state. In a mixed state, the quantum properties are indeed localized, but such a state cannot be used to start the computation on a quantum computer.

\section{Conclusions}

The representation of the state of the register in terms of $2^{n}$ components, which is the starting point of most quantum computing algorithms, is contingent on steering an initial state, which appears to be unrealizable. If the starting state cannot be realized, then such quantum computing models can only be taken to be mathematical constructs, not in accord with physical reality. 
If the cells of the register are independent quantum systems, then the uniqueness of the contents can be maintained. But in this case, the cells must be coupled for any useful computation to be possible. The couplings and the resultant entanglements form complications in the model beyond the scope of the standard quantum computing paradigm. This scenario departs from the usual one and the physical constraints necessary to be satisfied for this system to process superpositions of qubits need to be examined.

The reduction in the number of distinguishable states from $2^{n}$ components of the $n$ qubits to the $n+1$ distinguishable states of the coherent quantum system means that there is a corresponding reduction in entropy from $n$ to $\log _{2}(n+1)$. This means that energy equal to

$$
\left[n-\log _{2}(n+1)\right] k T \ln 2
$$

must be removed from the system [10] for a computation to proceed. But, as explained earlier, this computation cannot be based on a priori assignment of states to the individual qubits.

This analysis indicates that quantum statistical constraints need to be considered in the formulation of quantum algorithms and in their implementation. These constraints add to the difficulty of initialization of the state on the quantum register before the start of the computation.

We do not claim that these constraints will apply to all quantum computational schemes. This makes it important to look for such computational schemes where this is not a problem. Likewise, one needs to look for effective ways of preparing certain states of $n$-qubits. 


\section{References}

1. D. Deutsch, Proc. R. Soc. Lond. A 425, 73 (1989).

2. P.W. Shor, SIAM J. Computing 26, 1474 (1997), L.K. Grover, Physical Review Letters 79, 325 (1997).

3. A. Ekert and R. Jozsa, Rev. of Mod. Phys. 68, 733 (1996),

S. Bose et al, Phil.Trans.Roy.Soc.Lond. A356, 1823 (1998).

4. N. Gershenfeld and I.L. Chuang, Science 275, 350 (1997),

D.G. Cory et al, Proc. Natl. Acad. Science USA 94, 1634 (1997),

J.A. Jones et al, Nature 403, 869 (2000),

L.M.K. Vandersypen et al, Phys. Rev. Lett. 85, 5452 (2000).

5. S.L. Braunstein et al, Phys. Rev. Lett. 83, 1054 (1999).

6. S. Kak, Foundations of Physics 29, 267 (1999),

S. Kak, Information Sciences 128, 149 (2000).

7. A.K. Pati and S.L. Braunstein, Nature 400, 164 (2000).

8. D. Jonathan and M.B. Plenio, Phys. Rev. Lett. 83, 3566 (1999).

9. A. Peres, Quantum Theory: Concepts and Methods. Kluwer Academic, Dordrecht, 1995.

10. S. Kak, Foundations of Physics 26, 1375 (1996). 